\title{
Association of tricellulin expression with poor colorectal cancer prognosis and metastasis
}

\author{
JIN-XIU ZHANG ${ }^{*}$, MENG-BIN QIN*, ZHE YE, PENG PENG, SI-MAN LI, QIAN SONG, \\ LAN LIN, SHI-QUAN LIU, LI-HUA XIE, YE ZHU and JIE-AN HUANG \\ Department of Gastroenterology, The Second Affiliated Hospital of Guangxi Medical University, \\ Nanning, Guangxi Zhuang Autonomous Region 530007, P.R. China
}

Received April 6, 2020; Accepted August 20, 2020

DOI: $10.3892 /$ or.2020.7773

\begin{abstract}
Tricellulin is a tight-junction transmembrane protein that regulates cell-cell interactions. Altered tricellulin expression could promote tumor cell invasions and metastasis in human cancers. The present study assessed tricellulin expression in colorectal cancer tissues for any association with clinicopathological features of colorectal cancer patients and then investigated the underlying molecular events using quantitative proteomic analysis and in vitro experiments. Tissue samples from 98 colorectal cancer patients and 15 volunteers were collected for immunohistochemistry. Colorectal cell lines were used to overexpress or knockdown tricellulin expression in various assays. The data revealed that upregulated tricellulin expression was associated with lymph node and distant metastases and poor prognosis, while tricellulin overexpression promoted colorectal cancer cell migration and invasion in vitro. In contrast, tricellulin knockdown had positive effects on the tumor cells. Furthermore, TMT-LC-MS/MS and bioinformatics analyses revealed that tricellulin was involved in EMT and reduction of apoptosis through the $\mathrm{NF}-\kappa \mathrm{B}$ signaling pathway. These findings highlight for the first time the significance of tricellulin in colorectal cancer development and progression. Further study may validate tricellulin as a novel biomarker and target for colorectal cancer.
\end{abstract}

\section{Introduction}

Colorectal cancer accounts for approximately $10 \%$ of all tumors diagnosed each year and cancer-related deaths (1). It

Correspondence to: Professor Jie-An Huang, Department of Gastroenterology, The Second Affiliated Hospital of Guangxi Medical University, 166 Daxuedong Road, Nanning, Guangxi Zhuang Autonomous Region 530007, P.R. China

E-mail: hjagxmu@163.com

\section{${ }^{*}$ Contributed equally}

Key words: colorectal cancer, tricellulin, prognosis, tandem mass tag-liquid chromatography-mass spectrometry is the fourth most deadly cancer with approximately 900,000 colorectal cancer related-deaths annually worldwide (2). The risk factors of colorectal cancer include an unhealthy diet (especially excessive consumption of red and processed meats and alcohol), obesity, tobacco smoking, and lack of physical activity as well as genetic (familial adenomatous polyposis and hereditary non-polyposis colon cancer) and pre-cancer conditions such as inflammatory bowel diseases (Crohn's disease and ulcerative colitis) (3). Endoscopy is the most useful clinic tool for early detection and diagnosis of colorectal cancer while computed tomography (CT), magnetic resonance imaging (MRI), and positron-emission tomography (PET) are helpful for detecting colorectal cancer metastasis (3).

Treatment of colorectal cancer, like most other malignancies, comprises surgical resection of tumor lesions and adjuvant or neoadjuvant chemoradiotherapy or palliative care. These treatments depend on the tumor stage $(4,5)$, while recent immunotherapy and molecularly targeted therapy are also used to successfully treat colorectal cancer $(6,7)$. However, the prognosis of colorectal cancer remains unfavorable, especially at the advanced stages of disease (8-10). Therefore, a better understanding of colorectal carcinogenesis and molecular mechanisms could aid in more effectively preventing the start or progression of colorectal cancer clinically.

Tight junctions are the main structures that maintain intercellular connections for cell-cell adhesion, intercellular spaces, and conservation of solutions and other macromolecules through the paracellular pathway (11). To date, at least 40 different proteins have been identified that play roles in the tight junctions. Most of these proteins belong to the three major transmembrane protein groups: Occludin, claudins, and junction adhesion molecules (JAM) (12).

Accumulating evidence has revealed that aberrant expression of the tight junction proteins contributes to the development and metastasis of numerous epithelial-derived cancers (13). Tricellulin, also known as MARVELD2, was the first identified protein to be exclusively localized at the tri-epithelial junctions and to be revealed to regulate the junctional tension of epithelial cells (14) and provide a fulcrum for various epithelial stereo structures (15). The adhesion between epithelial cells is enhanced when tricellulin expression increases, whereas the tight junction function of these cells is lost when tricellulin is deleted (16). A previous study reported 
that nuclear tricellulin expression was associated with poorly differentiated pancreatic cancer and invasion of pancreatic cancer cells (17). To date, few studies have investigated the role of tricellulin in human cancers.

In the present study, first, tricellulin expression was analyzed in colorectal cancer tissues to determine its association with clinicopathological features of colorectal cancer patients and then the underlying molecular events were investigated via quantitative proteomic analysis and in vitro experiments. The aim of the present study was to provide novel and valuable information regarding the detection of tricellulin as a biomarker for colorectal cancer.

\section{Materials and methods}

Patients and tissue samples. The present study was approved by the Ethics Committee of the First Affiliated Hospital of Guangxi Medical University (Nanning, China) with the approval no. BBMCEC2012063 and was conducted in accordance with the guidelines of the Helsinki Declaration. The patient cohort of 98 colorectal cancer tissues and 15 normal tissues were collected from September 2013 and September 2014 at The First Affiliated Hospital of Guangxi Medical University where colorectal cancer was histologically diagnosed and staged according to the National Comprehensive Cancer Network (NCCN) colorectal cancer classifications (18). These 98 patients included 60 (61.2\%) males and 38 (38.8\%) females and their ages ranged from 25 to 83 years with a mean age of 56 years. There were 36 tumors located in the left-sided colon and 62 in the right-side colorectum. Four tumors were mucinous adenocarcinoma and 94 were tubular adenocarcinoma and there were 10 patients at stage I, 35 at stage II, 26 at stage III, and 27 at stage IV of the disease.

Immunohistochemistry (IHC). For IHC the sections (4-5 $\mu \mathrm{m})$ were deparaffinized in xylene and rehydrated in a series of ethanol solutions and then immunostained following the procedures of a previous study (19) with a primary antibody against human tricellulin (cat. no. SAB1306444; Sigma-Aldrich; Merck KGaA) at a dilution of 1:100. The immunoreactivity score (IRS) system as described in a previous study (20) was used to semi-quantify the immunostaining data, i.e., the staining intensity (SI) $\mathrm{x}$ the percentage of positive cells.

Cell culture, infection, and treatment. Human colorectal adenocarcinoma cell lines HT29, HCT116, RKO, SW620, Caco 2 and HCT- 8 and a normal human colon mucosal epithelial cell line NCM460 were obtained from the Cell Resource Center, Shanghai Institute of Biochemistry and Cell Biology at the Chinese Academy of Sciences (Shanghai, China). The cell lines had been validated by performing short tandem repeat (STR) profiling. The cells were cultured in RPMI-1640 medium (Corning, Inc.) supplemented with 10\% FBS (Biological Industries) in a humidified incubator containing $5 \% \mathrm{CO}_{2}$ at $37^{\circ} \mathrm{C}$.

A tricellulin shRNA (targeting sequences of 5'-GATGAG CAGATTGCCACATCA-3') was cloned into a pcDNA6. 3-EGFP vector (Invitrogen; Thermo Fisher Scientific, Inc.). The vector or negative control vector (lenti-EGFP-miR) was used to generate lentiviruses in 293 cells, and the lentiviruses were used to infect HCT116 cells and named as tricellulin knocked down cells (TRIC-KD) or negative control cells (NC). In addition, lentiviral vector carrying human tricellulin cDNA (lenti-tricellulin-IRES-EGFP) or vector-only was used to infect HCT116 cells for tricellulin-overexpression (TRIC-OE) in cells. The efficiency of tricellulin knockdown or overexpression was confirmed by RT-qPCR and western blotting. To determine the effects of tricellulin on regulation of the canonical $\mathrm{NF}-\kappa \mathrm{B}$ pathway, we treated these stable cells or parental cells (CON) with $50 \mathrm{ng} / \mathrm{ml}$ TNF- $\alpha$ (R\&D Systems) or $100 \mu \mathrm{M}$ pyrrolidinecarbodithioic acid (PDTC; Sigma-Aldrich; Merck $\mathrm{KGaA}$ ) for $24 \mathrm{~h}$ for subsequent experiments.

Immunofluorescence and phalloidin staining. Fresh colorectal cancer tissues were cryosectioned and fixed in $4 \%$ paraformaldehyde and then immunostained following the procedures of a previous study (21) with a primary antibody against tricellulin (product no. 48-8400; Invitrogen; Thermo Fisher Scientific, Inc.) at a dilution of 1:200 at $4^{\circ} \mathrm{C}$ overnight. Subsequently, the sections were washed and incubated with the respective secondary antibody (Alexa Fluor ${ }^{\circledR} 594$ goat anti-rabbit; product no. 8889S; 1:1,000; Cell Signaling Technology, Inc.) and 4',6-diamidino-2-phenylindole (DAPI) (Wuhan Boster Biological Technology, Ltd.).

To visualize the cell actin cytoskeleton, FITC-Phalloidin was utilized to stain HCT116 cells (NC, TRIC-KD, and TRIC-OE). In brief, cells $\left(1 \times 10^{4}\right)$ were seeded onto coverslips and grown overnight and then fixed and permeabilized in the same way as the cryosections. The cells were next incubated with the Alexa Fluor ${ }^{\circledR} 488$ Phalloidin working solution (Beijing Solarbio Science \& Technology, Inc.) for $30 \mathrm{~min}$ at room temperature in the dark and then with DAPI for $10 \mathrm{~min}$. Images were obtained from the stained cells with a fluorescence Olympus BX53F microscope (magnification, x600; Olympus Corporation).

$R N A$ isolation and reverse transcription $(R T)$-quantitative $(q) P C R$. Total cellular RNA was isolated from cells using the Eastep $^{\text {TM }}$ Super Total RNA Extraction Kit and reversely transcribed into cDNA using a Reverse Transcription Kit (both from Promega Corporation). qPCR was performed using the Power SYBR Green Master Mix (Promega Corporation) on an 7500 ABI Real-time PCR system (Applied Biosystems; Thermo Fisher Scientific, Inc.). The primers used were tricellulin forward, 5'-CCAGCTATAGCGCCAGATCTCAA-3' and reverse, 5'-CAGACACCGGCTTATCCCATTC-3'; and GAPDH forward, 5'-GCACCGCAAGGCTGAGAAC-3' and reverse, 5'-TGGTGAAGACGCCAGTGGA-3', which were all obtained from Takara Bio, Inc.. The cycling parameters were as follows: Denaturing at $95^{\circ} \mathrm{C}$ for $10 \mathrm{~min}, 40$ cycles of denaturing at $95^{\circ} \mathrm{C}$ for $15 \mathrm{sec}$, primer annealing at $60^{\circ} \mathrm{C}$ for $1 \mathrm{~min}$ and extension temperature at $95^{\circ} \mathrm{C}$ for $15 \mathrm{sec}$; final extension at $60^{\circ} \mathrm{C}$ for $15 \mathrm{sec}$ and final denaturing at $95^{\circ} \mathrm{C}$ for $15 \mathrm{sec}$. Results were quantified by using the $2^{-\Delta \Delta \mathrm{Cq}}$ method (22).

Western blotting. Cells were lysed using the RIPA lysis buffer (Beyotime Institute of Biotechnology) and quantified by using the bicinchoninic acid (BCA) protein assay kit (Beyotime Institute of Biotechnology). Equal amounts (30 $\mu \mathrm{g} /$ lane) of 
protein samples were separated in 10 or $12 \%$ sodium dodecyl sulfate-polyacrylamide gel electrophoresis (SDS-PAGE) gels and transferred onto polyvinylidene fluoride membranes (PVDF; EMD Millipore).

Western blotting was performed according to our previous study (23); after being blocked in a 5\% skim milk for $1 \mathrm{~h}$ at the room temperature, the membranes were incubated with a primary antibody against GAPDH (cat. no. 60004-1-Ig; 1:3,000), MMP2 (cat. no. 10373-2-AP; 1:800), vimentin (cat. no. 10366-1-AP; 1:500), Snail (cat. no. 13099-1-AP; 1:500), caspase-3 (product no. 19677-1-AP; 1:800) (all from ProteinTech Group, Inc.), tricellulin (cat. no. 48-8400; 1:800; Invitrogen; Thermo Fisher Scientific, Inc.), p65 (product no. 8242S; 1:1,000), p-p65 Ser536 (product no. 3033; 1:1,000), E-cadherin (product no. 3195; 1:1,000), survivin (product no. 2808S; 1:1,000), or Bcl-2 (product no. 15071S; 1:1,000) (all from Cell Signaling Technology, Inc.) overnight at $4^{\circ} \mathrm{C}$. Subsequently, the membranes were incubated with a secondary antibody (1:10,000; LI-COR IRDye ${ }^{\circledR}$ 680RD Goat anti-Rabbit P/N 926-68071 or LI-COR IRDye ${ }^{\circledR}$ 680RD Goat anti-Mouse $\mathrm{P} / \mathrm{N}$ 926-68070; LI-COR Biosciences) at room temperature for $1 \mathrm{~h}$. The protein bands were quantified by using Odyssey infrared imaging (LI-COR Biosciences). The final data were semi-quantification was carried out using ImageJ software (version 1.50i; National Institutes of Health).

Transwell migration and invasion assays. The cells $\left(1 \times 10^{5}\right)$ were added to the upper chamber of a Transwell $(8-\mu \mathrm{m}$ pores) with noncoated membranes (migration assay) or Matrigel-coated Transwell chambers (invasion assay) with serum-free medium, while a cell culture medium containing $20 \%$ FBS was added to the bottom chambers. After 24 or $48 \mathrm{~h}$ culture, the migrated or invasive cells at the bottom of the membrane were fixed in $4 \%$ methanol for $20 \mathrm{~min}$ at room temperature, and stained using $0.1 \%$ crystal for $10 \mathrm{~min}$ at room temperature, and counted from five-randomly selected fields under the 100X objective of an inverted microscope (Nikon Corporation).

Hoechst 33258 staining. Cells were seeded into a 24-well flat bottom plate at a density of $1 \times 10^{5}$ cells/well and grown for $24 \mathrm{~h}$. Next, the cells were washed with phosphate-buffered saline (PBS), fixed in 4\% methanol, and incubated with Hoechst 33258 stain solution (Beijing Solarbio Science \& Technology, Inc.) for $10 \mathrm{~min}$ at room temperature. The cell apoptotic morphology was observed under an Olympus BX53F fluorescence microscope (magnification, x600).

Tandem mass tag (TMT)-labeling and liquid chromatographymass spectrometry (LC-MS/MS). TMT-LC-MS/MS was performed by R\&S Biotechnology Co., Ltd.. In brief, total protein was extracted from cells and processed in a 6-plex TMT labeling kit (Thermo Fisher Scientific, Inc.) according to a procedure of a previous study (24). Fractionation was then carried out by basic $\mathrm{pH}$ reverse-phase liquid chromatography with fraction combining according to a previous study (25). The separated samples were loaded onto the Nano-Aquity UPLC system (Waters Corporation) and the resulting peptide mixture was loaded onto a trap column (2.1x150 mm X Bridge BEH300; Waters Corporation) attached to an analytical column
(ZORBAX 300SB-C18 column, $5 \mu \mathrm{m}, 300 \AA$ A , 0.1x150 mm; Microm for mass-spectrometer analysis (26). The linear gradient was from 2\% D to $80 \%$ D in 90 min (solution D: $0.1 \%$ formic acid in ACN) and the triple TOF $5600 \mathrm{MS}$, switched between MS and serial (MS/MS) acquisition. The MS data were acquired using a spectral accumulation time of $250 \mathrm{msec}$ in the mass range of 350-1,300 m/z. The scanning tandem mass spectrometry was from $100-1250 \mathrm{~m} / \mathrm{z}$ with rolling collision energy. The 20 strongest precursors were selected according to the procedure of a previous study (26).

Identification of differentially expressed proteins and enrichment analysis in tricellulin overexpression (TRIC-OE) or NC cells. The data were then analyzed using the free online platform of Majorbio I-Sanger Cloud Platform (www.i-sanger. com). The ratio cutoff values were set to 1.2 and 0.8 for fold-change and more than two unique peptides were used for protein identification. A P-value $<0.05$ was considered as the threshold to identify significant changes. We then performed Gene Ontology (GO) (27) and used the Kyoto Encyclopedia of Genes and Genomes (KEGG) (28) database to identify the functions or interactions between these differentially expressed proteins (DEPs) in colorectal cancer cells.

Protein-protein interaction (PPI) network analysis. A PPI network was constructed using a Search Tool [STRING (29); http://string.embl.de/]. A confidence score of 0.4 was set as the cut-off criterion and the DEPs with connection numbers 10 were considered as hub genes. The top 10 hub nodes with higher degrees were screened using $\mathrm{R}$ (version 3.6.0, https://cran.r-project.org/).

Statistical analysis. All data were presented as the means \pm SD and statistically analyzed using the SPSS v17.0 software package (IBM Corp.) and GraphPad Prism v7.0 (GraphPad Software, Inc.). The association between tricellulin expression and clinicopathological characteristics of colorectal cancer patients was analyzed using the Chi-squared $\left(\chi^{2}\right)$ test, while the survival rates stratified by tricellulin expression were calculated by Kaplan-Meier curves and the log-rank test. Unpaired Student's t-tests and one-way ANOVA tests were used to determine statistical significance between two groups or among more than two groups, respectively. After a one-way ANOVA test was performed, for post hoc evaluation, Tukey's HSD test was used. A P-value $<0.05$ was considered to indicate a statistically significant difference.

\section{Results}

Tricellulin upregulation and association with poor colorectal cancer prognosis. Tricellulin expression was analyzed in 98 cases of colorectal cancer tissues vs. 15 normal mucosae and the results revealed that tricellulin expression was significantly upregulated in colorectal cancer tissues compared to normal mucosae (Fig. 1A). Tricellulin was mainly expressed in the cytoplasm or nucleus in colorectal cancer tissues, which was quite different from that of normal mucosae in the cytoplasm (Fig. 1B). Of these 98 cancer cases, 51 (52.0\%) had tricellulin overexpression vs. 2 (13.3\%) with tricellulin expression among the 15 normal mucosae (Fig. 1C). 
A

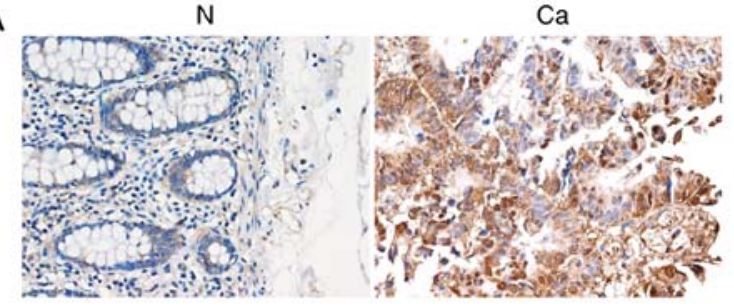

C

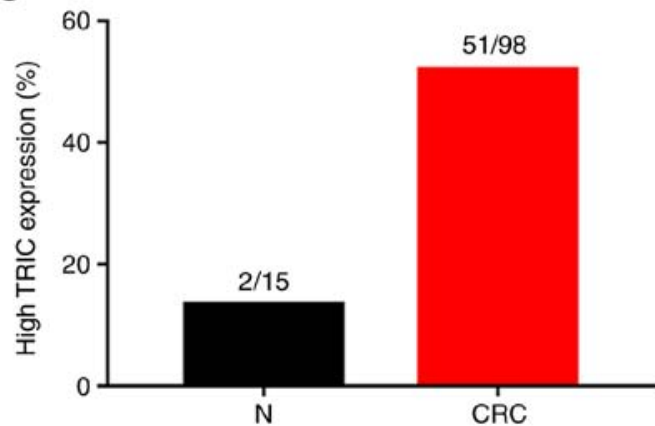

B
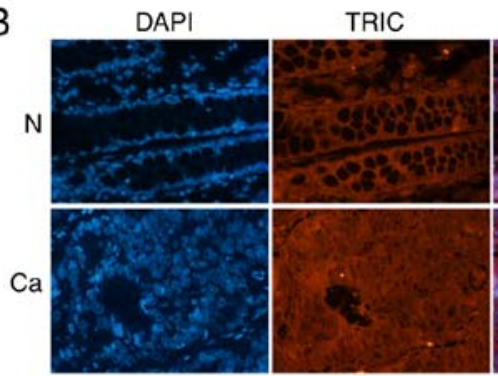

Merged

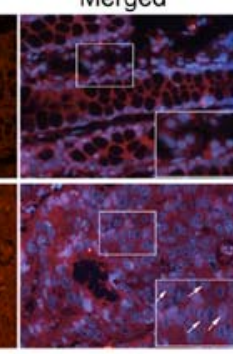

D

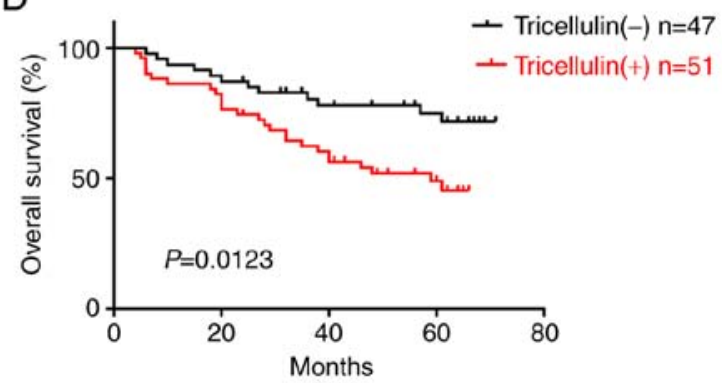

Figure 1. Tricellulin expression and association with poor survival of colorectal cancer patients. (A) IHC. Tricellulin expression was significantly higher in colorectal cancer tissues than in normal colorectal mucosae. Magnification, $x 400$. (B) Immunofluorescence staining of tricellulin in colorectal cancer tissues and normal colorectal mucosae. Magnification, x400. (C) Summarized data of IHC. The data revealed that 51 (52.0\%) colorectal cancer tissues had tricellulin overexpression, whereas 2 (13.3\%) normal mucosae had tricellulin expression. (D) Kaplan-Meier curves. Tricellulin expression is associated with poor OS of colorectal cancer patients $(\mathrm{n}=98)$. IHC, immunohistochemistry; OS, overall survival; N, normal; Ca, cancerous; TRIC, tricellulin; CRC, colorectal cancer.

Then, the association of tricellulin expression with clinicopathological characteristics of colorectal cancer patients was assessed and it was revealed that tricellulin expression (52.0\%; 51/98) was associated with tumor distant metastasis (M stage) and advanced tumor-lymph node metastasis (TNM stage) (Table I), but not with the sex and age, tumor size, and $\mathrm{N}$ stage of patients. The Kaplan-Meier curves and the long rank test revealed that the upregulated tricellulin expression was associated with shorter overall survival (OS; $\mathrm{P}=0.012$; Fig. 1D).

Effects of tricellulin knockdown and overexpression on regulation of colorectal cancer cell malignant phenotypes in vitro. To assess the effects of tricellulin knockdown and overexpression on regulation of colorectal cancer cell malignant phenotypes in vitro, tricellulin expression was first analyzed in human colorectal adenocarcinoma cell lines HT-29, HCT116, RKO, SW620, Caco2, and HCT8 as well as a normal human colon mucosal epithelial cell line, NCM460. It was revealed that the level of tricellulin protein was higher in HT29, HCT116, and SW620 cells than in other all cancerous and normal cells (Fig. 2A). We therefore selected HCT116 with moderate endogenous expression of tricellulin for stable tricellulin knockdown or overexpression. Tricellulin knockdown (TRIC-KD) or overexpression (TRIC-OE) was confirmed by RT-qPCR and western blotting (Fig. 2B-E).

Next, it was determined that TRIC-OE significantly increased HCT116 cell migration and invasion capacities, whereas TRIC-KD reduced tumor cell migration and invasion (Fig. 3A-C). The FITC-Phalloidin staining revealed that tricellulin regulated pseudopodium formation and cytoskeletal reorganization; downregulation of tricellulin expression notably inhibited the length and number of pseudopodia and stress fibers. In contrast, upregulation of tricellulin expression
Table I. Association of tricellulin expression with clinicopathological features from colorectal patients.

\begin{tabular}{lccc}
\hline & \multicolumn{2}{l}{ Tricellulin expression } & \\
\cline { 2 - 3 } Characteristics & Negative & Positive & P-value \\
\hline Sex & & & 0.202 \\
Male & 33 & 28 & \\
Female & 16 & 23 & \\
Age (years) & & & 0.817 \\
$\geq 65$ & 13 & 12 & \\
$<65$ & 37 & 38 & \\
Treatment & & & 0.249 \\
Surgery & 21 & 17 & \\
Comprehensive & 26 & 34 & \\
N stage & & & 0.220 \\
N0 & 30 & 25 & \\
N1+N2 & 19 & 26 & \\
M stage & & & 0.025 \\
M0 & 39 & 30 & \\
M1 & 10 & 21 & \\
TNM stage & & & 0.004 \\
I+II & 30 & 17 & \\
III+IV & 19 & 34 & \\
\hline
\end{tabular}

M, tumor distant metastasis; TNM, tumor-lymph node metastasis.

increased the length and number of pseudopodia and stress fibers (Fig. 3D). 


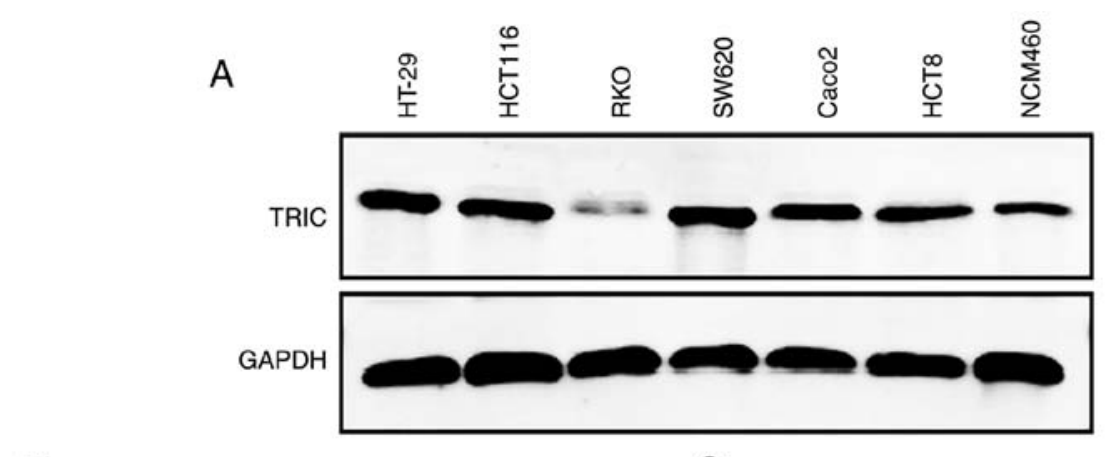

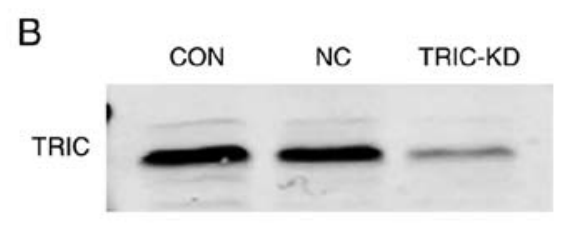
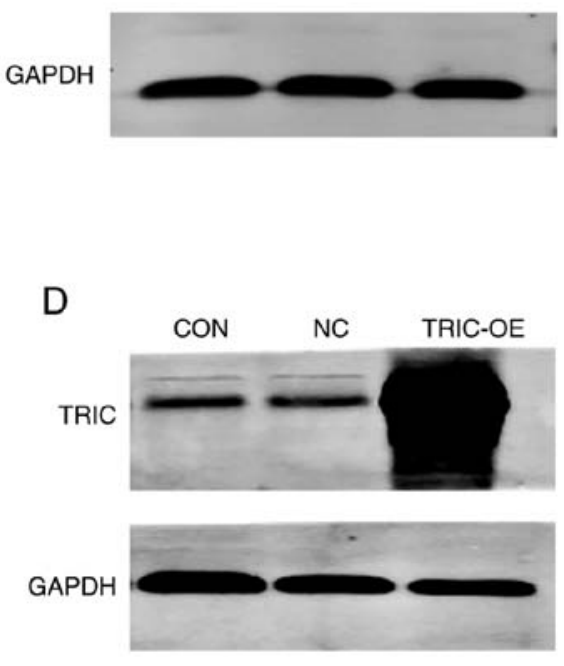

C
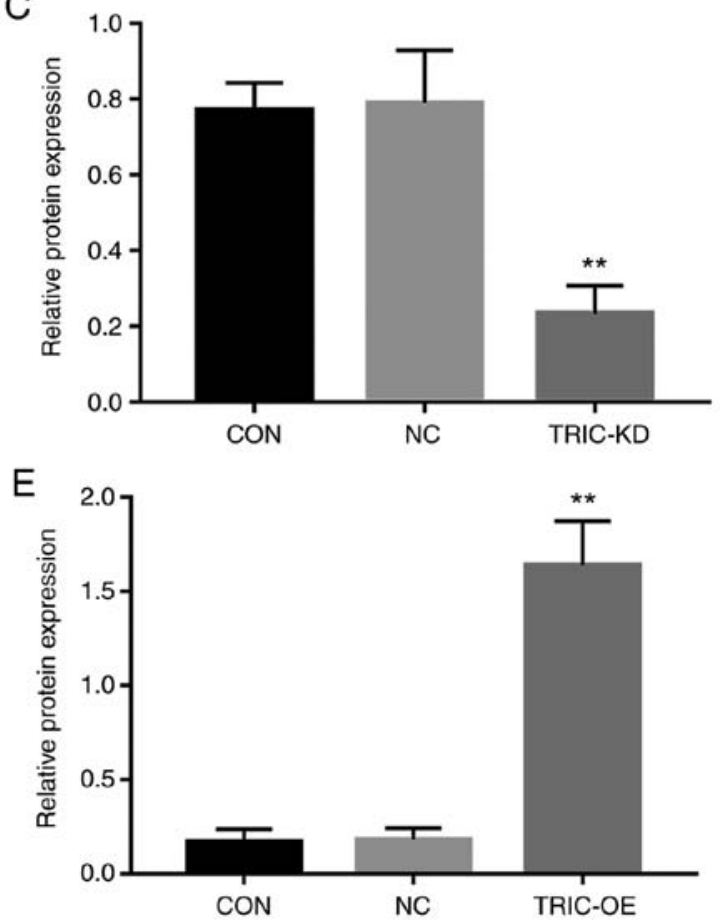

Figure 2. Stable knockdown or overexpression of tricellulin in colorectal cancer cells. (A) Western blotting. Tricellulin expression was assessed in a panel of colorectal cancer cell lines and a normal human colon mucosal epithelial cell line NCM460 using western blotting. (B and C) Western blotting and RT-qPCR analysis for TRIC-KD. GAPDH was used as a reference. (D and E) Western blotting and RT-qPCR analysis for TRIC-OE. GAPDH was used as the reference. RT-qPCR, reverse transcription-quantitative PCR; TRIC-KD, tricellulin knockdown; TRIC-OE, tricellulin overexpression; TRIC, tricellulin; CON, control; $\mathrm{NC}$, negative control.

Tricellulin regulation of differentially expressed proteins in colorectal cancer cells by quantitative proteomic analysis. To elucidate the potential molecular mechanisms underlying tricellulin, quantitative proteomic analysis was performed to profile DEPs in the TRIC-OE group vs. the NC group. We identified a total of 6,957 unique proteins in the TRIC-OE group and 193 were DEPs vs. the NC group, in which 63 were upregulated and 130 were downregulated. The DEPs volcano plots are presented in Fig. 4A.

To determine their biological and functional properties in the TRIC-OE compared to the NC group, GO was performed and the KEGG database was used. The GO data revealed that tricellulin could regulate the biological process (BP), molecular function (MF), and cellular component (CC). These DEPs were significantly enriched in regulation of the ventricular cardiac muscle cell action potential, peptidyl-cysteine S-nitrosylation, lateral sprouting from an epithelium, epithelial-mesenchymal transition (EMT), and phosphatidylinositol 3-kinase signaling (Fig. 4B). The KEGG analysis revealed that DEPs were mainly enriched in nicotine addiction, basal cell carcinoma, Wnt signaling pathway, circadian entrainment, and pathways in cancer (Fig. 4C).

The PPI network data with the confidence score set at 0.4 revealed that the hub genes with higher degrees included UBE2D1, DTL, TIMP1, CREB1, CYCS, PIK3CA, CENPH, COL1A1, COMMD1, and PTTG1 (Fig. 4D).

Tricellulin regulation of colorectal cancer cell EMT via the canonical nuclear factor- $\kappa$-light-chain-enhancer of activated Bcells $(N F-\kappa B)$. EMT, a key developmental regulatory program, plays a critical role in the promotion of cancer progression and metastasis in human carcinomas (30). In our previous study, we demonstrated that the NF- $\kappa \mathrm{B}$ signaling was closely associated with colorectal cancer cell EMT (31). Thus, we further determined the role of the $\mathrm{NF}-\kappa \mathrm{B}$ pathway in mediating the tricellulin oncogenic activities in colorectal cancer cells. The expression of p65, p-p65, mesenchymal markers (N-cadherin, Snail and vimentin), and an epithelial marker (E-cadherin) was analyzed in HCT116 cells after tricellulin overexpression of knockdown. The data revealed that tricellulin overexpression 


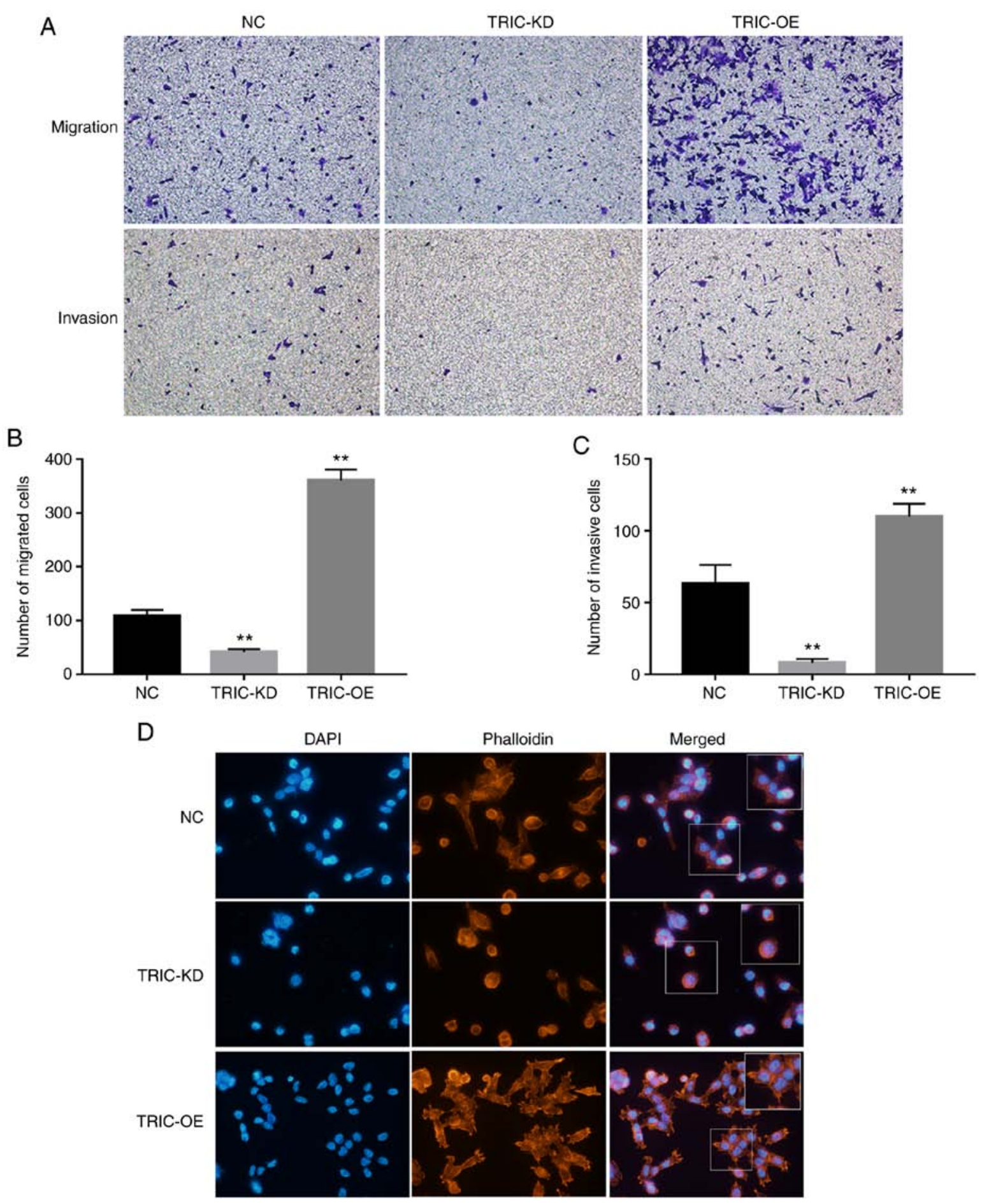

Figure 3. Effects of tricellulin overexpression and knockdown on regulation of colorectal cancer cell invasion and gene expression. (A-C) Transwell assay. Stable HCT116 cells were grown and subjected to Transwell migration and invasion assay (magnification, $\mathrm{x} 200$ ). The graphs are summarized data of the assays of B and C. (D) FITC-Phalloidin staining. HCT116 cells were grown and stably infected with lentiviruses carrying tricellulin cDNA (TRIC-OE) or shRNA (TRIC-KD) or NC and then subjected to FITC-Phalloidin staining. TRIC-KD, tricellulin knockdown; TRIC-OE, tricellulin overexpression; NC, negative control; TRIC, tricellulin.

upregulated the levels of vimentin and Snail but downregulated E-cadherin, which was not completely inhibited by an NF- $\mathrm{KB}$ inhibitor PDTC (Fig. 5A). In contrast, knockdown of tricellulin expression decreased vimentin and Snail, but increased E-cadherin, which was also not completely activated by an NF- $\kappa$ B activator TNF- $\alpha$ (Fig. 5B). These results indicated that tricellulin may regulate the EMT in colorectal cancer cell through the canonical NF- $\mathrm{kB}$ signaling pathways.
Tricellulin regulation of HCT116 cell apoptosis in vitro. Hoechst 33258 staining and western blotting were first performed to further assess the role of tricellulin in apoptosis. The present data revealed that the apoptotic cells had significant nuclear condensation and morphological changes in TRIC-KD cells compared to the negative control group (NC). Conversely, tricellulin overexpression (TRIC-OE) had normal nuclear morphology (Fig. 6A). Assessment of the 
A
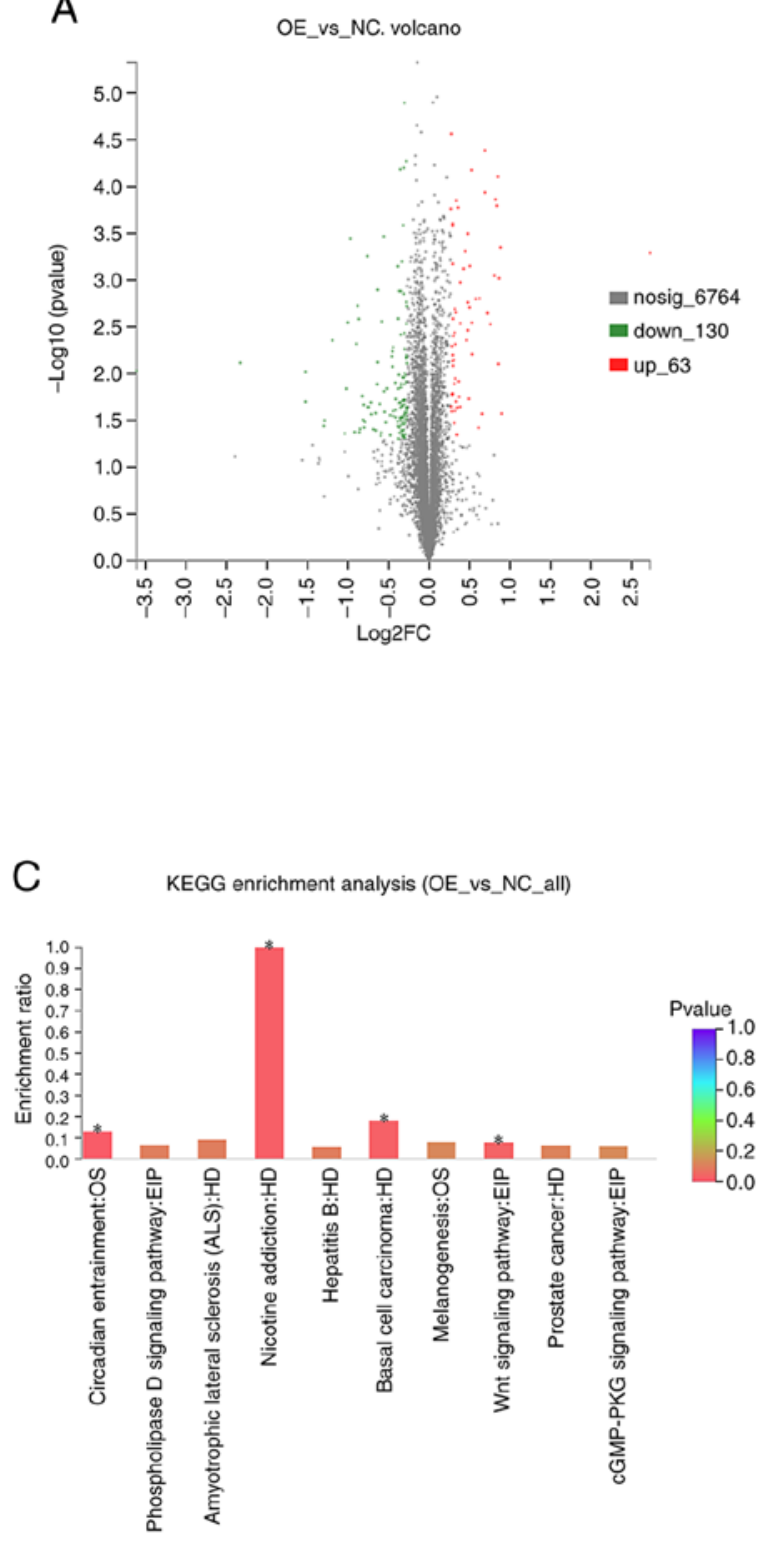

B

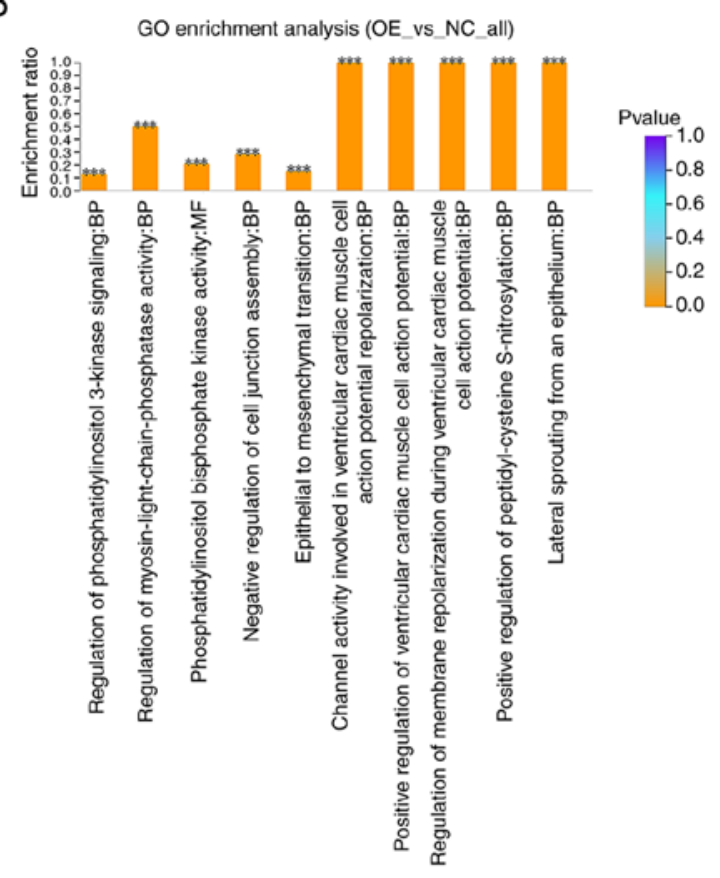

D

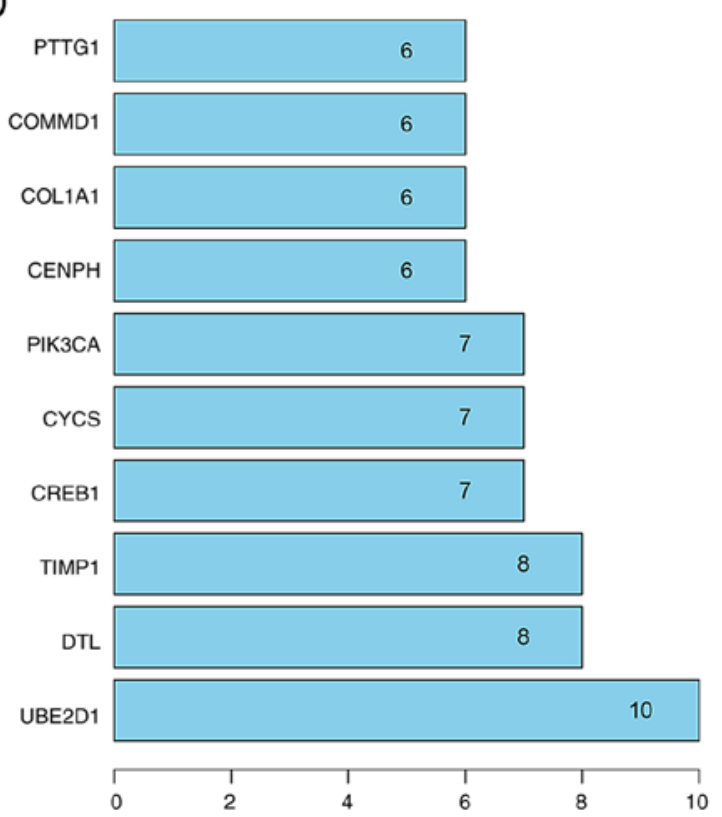

Figure 4. DEPs in TRIC-OE HCT116 cells or NC identified by TMT-LC-MS/MS and bioinformatics analysis. (A) DEPs with a cutoff value of 1.2 or 0.8 for fold-change and $\mathrm{P}<0.05$ were plotted in the volcano plot. (B) GO data. The top $10 \mathrm{GO}$ terms based on the GO analysis of NC vs. TRIC-OE HCT116 cells. (C) The KEGG pathway analysis. The top 10 signaling pathways enriched based on the KEGG pathway analysis of NC vs. TRIC-OE HCT116 cells. (D) The PPI network analysis. The top 10 hub nodes were shown with higher degrees. Differentially expressed proteins; TRIC-OE, tricellulin overexpression; NC, negative control; TMT-LC-MS/MS, tandem mass tag-labeling and liquid chromatography-mass spectrometry; GO, Gene Ontology; KEGG, Kyoto Encyclopedia of Genes and Genomes; PPI, protein-protein interaction.

levels of apoptosis-related proteins revealed that tricellulin overexpression reduced caspase- 3 and increased Bcl- 2 and survivin levels, whereas knockdown of tricellulin expression reduced Bcl-2 and survivin levels and enhanced caspase-3 levels (Fig. 6B and C). These data indicated that tricellulin enhanced tumor cell survival.

\section{Discussion}

Aberrant expression and functions of the tight junction-related proteins contribute to the development of various human cancers (32). Tricellulin localizes at tricellular tight junctions as a member of the tight-junction-associated MARVEL protein (TAMP) family (33). Nuclear or cytoplasmic tricellulin expression was associated with pancreatic cancer cell invasion and metastasis as well as a poor prognosis (17).

To date, there is no study revealing the role of tricellulin in colorectal cancer. Thus, the present study was the first, to the best of our knowledge, to demonstrate tricellulin overexpression in colorectal cancer tissues with both cytoplasmic and nuclear localizations as well as tricellulin expression associated with colorectal cancer lymph node and distant metastasis 


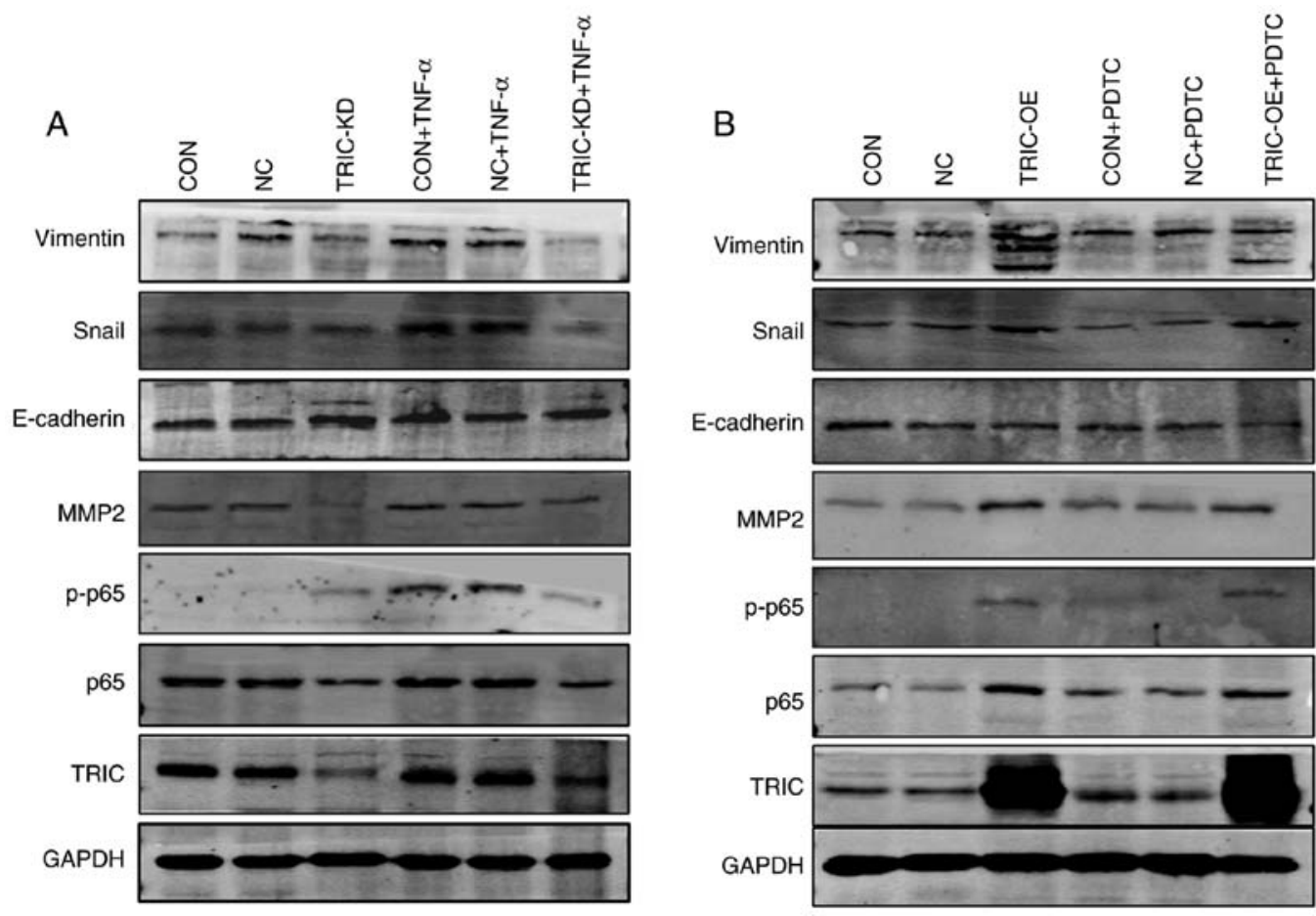

Figure 5. Effect of TRIC-KD or TRIC-OE on the regulation of colorectal cancer cell EMT and NF- $\kappa B$ expression. (A) Western blotting. Expression of the EMT markers and canonical NF- $\mathrm{KB}$ signaling pathways markers was analyzed in HCT116 cells after TRIC-KD with or without TNF- $\alpha$ treatment. (B) Western blotting. Expression of the EMT markers and canonical NF- $\mathrm{BB}$ signal pathways markers was assessed using western blotting in HCT116 cells after TRIC-OE with or without PDTC treatment. TRIC-KD, tricellulin knockdown; TRIC-OE, tricellulin overexpression; EMT, epithelial-mesenchymal transition; NF- $\mathrm{BB}$, nuclear factor-k-light-chain-enhancer of activated B cells; PDTC, pyrrolidinecarbodithioic acid; CON, control; NC, negative control; TRIC, tricellulin.

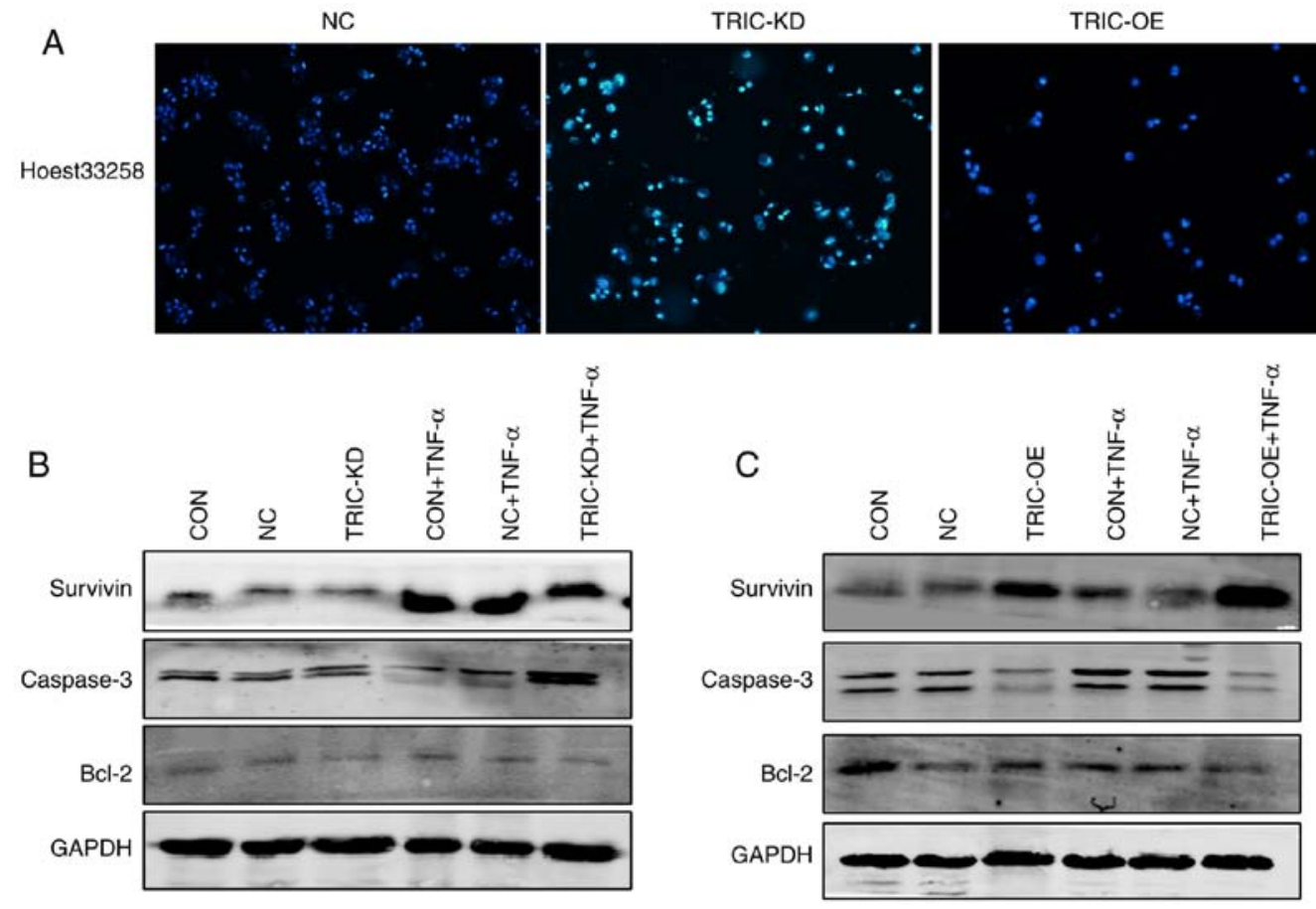

Figure 6. Effect of tricellulin knockdown or overexpression on regulation of colorectal cancer HCT116 cell apoptosis. (A) Hoechst 33258 staining. Apoptosis was detected by Hoechst 33258 staining. Magnification, x200. (B) Western blotting. Expression of the apoptosis markers was detected using western blotting in HCT116 cells after TRIC-KD with or without TNF- $\alpha$ treatment. (C) Western blotting. Expression of the apoptosis markers was assessed using western blotting in HCT116 cells after TRIC-OE with or without PDTC treatment. TRIC-KD, tricellulin knockdown; TRIC-OE, tricellulin overexpression; PDTC, pyrrolidinecarbodithioic acid; CON, control; NC, negative control; TRIC, tricellulin.

and poor prognosis, which is consistent with those occurring in pancreatic cancer (17).
Using in vitro functional assays, it was revealed that knockdown of tricellulin expression inhibited colorectal cancer cell 
invasion and migration, whereas tricellulin overexpression enhanced tumor cell invasion and migration. Our later experiments also showed that tricellulin expression upregulated actin and cytoskeletal reorganization and EMT in HCT116 cells. In fact, EMT drives carcinoma progression and plays an essential role in metastasis (34). Moreover, multiple signaling pathways are involved in EMT development (35); for example, the NF- $\kappa \mathrm{B}$ signaling is involved in EMT regulation $(31,36)$.

The present study further confirmed the involvement of the NF- $\kappa \mathrm{B}$ signaling pathway in the regulation of tricellulin-induced colorectal cancer cell EMT in vitro. In addition, the $\mathrm{GO}$ data revealed that tricellulin could regulate the $\mathrm{BP}$, MF, CC, and EMT, while the PPI network data revealed that the hub genes included UBE2D1, DTL, TIMP1, CREB1, CYCS, PIK3CA, CENPH, COL1A1, COMMD1, and PTTG1. However, further studies are required to fully reveal the functions and regulations of tricellulin in colorectal cancer development and progression. In fact, the cell tight-junction helps to hold cells together and enable cell-cell communication. It also acts a barrier so that cell polarity is maintained and it prevents the lateral diffusion of integral membrane proteins and molecules and loss of ions (3). For example, knockdown of tricellulin expression using RNAi compromised the epithelial barrier and tricellular contacts and disorganized the bicellular tight-junction (37).

It remains unknown how tricellulin overexpression dysregulated cancer cell invasion and metastasis, including colorectal cancer. A previous study revealed that nuclear tricellulin possessed an oncogenic activity in pancreatic cancer (17). Knockdown of the tricellular tight junction protein lipolysis-stimulated lipoprotein receptor (LSR) expression induced tricellulin relocalization from the tricellular region to the bicellular region at the membrane to promote endometrial cancer cell proliferation, migration, and invasion (38). However, tricellulin expression revealed a significant negative correlation with differentiation in pancreatic ductal adenocarcinoma (38).

Tricellulin expression was associated with favorable prognosis in human hepatoblastoma patients (39). In normal gastric mucosa, tricellulin was localized at the tricellular tight junction, whereas tricellulin was distributed in the cytoplasm of gastric cancer cells (40). Transduction of Snail reduced the level of tricellulin and E-cadherin but increased vimentin and $\mathrm{N}$-cadherin, thus indicating that suppression of tricellulin expression mediated Snail-induced EMT in human gastric cancer cells (40). However, another previous study revealed tricellulin overexpression and an association with poor prognosis of hepatocellular carcinoma (HCC), although higher grades of intrahepatic cholangiocarcinoma revealed decreases in tricellulin expression, which was correlated with poor prognosis (41). The present data are consistent with these latter studies.

Collectively, tricellulin expression and cellular localization have different roles in different human cancers $(17,38,40)$. Furthermore, $\mathrm{NF}-\kappa \mathrm{B}$ is a protein complex and functions to regulate gene transcription, cytokine production, and cell survival $(42,43)$. In human cancers, $N F-\kappa B$ activity is frequently induced for tumor cell proliferation and survival $(43,44)$.

In the present study, it was revealed that tricellulin overexpression upregulated levels of vimentin and Snail but downregulated E-cadherin, which was not completely inhibited by an NF- $\mathrm{NB}$ inhibitor PDTC. In contrast, knockdown of tricellulin expression decreased vimentin and Snail, but increased E-cadherin, which was also not completely activated by an NF- $\kappa \mathrm{B}$ activator TNF- $\alpha$, thus indicating that tricellulin may regulate the EMT in colorectal cancer cells through the canonical NF- $\kappa \mathrm{B}$ signaling pathway. In fact, a previous study revealed that upregulation of TRIC, p-JNK and p-IкB was observed after treatment of human pancreatic cancer cell lines HPAC with IL-1 $\beta$, TNF- $\alpha$ and IL-1 $\alpha$ for $24 \mathrm{~h}$, whereas change in phospho-I $\kappa \mathrm{B}$ was inhibited by $\mathrm{JNK}$ and $\mathrm{NF}-\kappa \mathrm{B}$ inhibitors. However, after treating hTERT-transfected human pancreatic duct epithelial cells (hTERT-HPDE cells) with IL-1 $\beta$, TNF- $\alpha$, and IL-1 $\alpha$ for $24 \mathrm{~h}$, tricelluin expression was upregulated (45).

To date, no studies have shown whether tricellulin regulated EMT, thereby affecting invasion and metastasis of colorectal cancer via the NF- $\kappa \mathrm{B}$ signaling pathway. A previous study reported that $\mathrm{NF}-\kappa \mathrm{B}$ induced the expression of anti-apoptotic genes, inhibited the expression of apoptotic proteins, and regulated members of the Bcl-2 family of apoptosis regulators, while tumor cells may also rely on the $\mathrm{NF}-\kappa \mathrm{B}$ pathway to escape from apoptosis, which has been demonstrated to be one of the important markers of cancer (46). Thus, the present study provided such data, although further studies are required to further reveal the exact underlying mechanisms.

In summary, the present study demonstrated that tricellulin expression was associated with colorectal cancer metastasis and poor prognosis, while tricellulin expression promoted colorectal cancer cell invasion and EMT in vitro. The present data support the theory that tricellulin is involved in colorectal cancer metastasis, and further studies will validate the detection of tricellulin as a novel marker for prediction of colorectal cancer prognosis. Mechanistically, tricellulin could activate the canonical NF- $\kappa \mathrm{B}$ signaling pathway for promotion of colorectal cancer cell EMT and inhibition of tumor cell apoptosis.

\section{Acknowledgements}

Not applicable.

\section{Funding}

The present study was supported in part by grants from the Natural Science Foundation of China (grant no. 81760516), the 2018 Innovation Project of Guangxi Graduate Education (grant no. YCBZ2018046), and the Nature Science Foundation of Guangxi (grant no. 2019GXNSFAA185030).

\section{Availability of data and materials}

All data generated or analyzed in the current study are included in this publication and are available on reasonable request.

\section{Authors' contributions}

JAH, JXZ and MBQ conceived and designed the experiments. JXZ and ZY wrote the manuscript. ZY, SML, PP, and QS performed the experiments. LL, LHX, YZ and SQL analyzed the data. All authors read and approved the manuscript and agree to be accountable for all aspects of the research in 
ensuring that the accuracy or integrity of any part of the work are appropriately investigated and resolved.

\section{Ethics approval and consent to participate}

The present study was approved by the Ethics Committee of the First Affiliated Hospital of Guangxi Medical University (Guangxi, China) with the approval no. BBMCEC2012063 and performed in accordance with the guidelines of the Declaration of Helsinki. Written informed consent was obtained from each patient.

\section{Patient consent for publication}

Not applicable.

\section{Competing interests}

The authors declare that they have no competing interests.

\section{References}

1. Bray F, Ferlay J, Soerjomataram I, Siegel RL, Torre LA and Jemal A: Global cancer statistics 2018: GLOBOCAN estimates of incidence and mortality worldwide for 36 cancers in 185 countries. CA Cancer J Clin 68: 394-424, 2018.

2. Dekker E, Tanis PJ, Vleugels JL, Kasi PM and Wallace MB: Colorectal cancer. Lancet 394: 1467-1480, 2019.

3. Fujita T: Colorectal cancer. Lancet 376: 331-332, 2010.

4. Stein A, Atanackovic D and Bokemeyer C: Current standards and new trends in the primary treatment of colorectal cancer. Eur J Cancer 47 (Suppl 3): S312-S314, 2011.

5. Fakih MG: Metastatic colorectal cancer: Current state and future directions. J Clin Oncol 33: 1809-1824, 2015.

6. Boland PM and Ma WW: Immunotherapy for colorectal cancer. Cancers (Basel) 9: 50, 2017.

7. Syn NL, Teng MWL, Mok TSK and Soo RA: De-novo and acquired resistance to immune checkpoint targeting. Lancet Oncol 18: e731-e741, 2017.

8. Brenner $\mathrm{H}$ and Chen $\mathrm{C}$ : The colorectal cancer epidemic: Challenges and opportunities for primary, secondary and tertiary prevention. Br J Cancer 119: 785-792, 2018.

9. Wang W, Kandimalla R, Huang H, Zhu L, Li Y, Gao F, Goel A and Wang X: Molecular subtyping of colorectal cancer: Recent progress, new challenges and emerging opportunities. Semin Cancer Biol 55: 37-52, 2019.

10. Zacharakis M, Xynos ID, Lazaris A, Smaro T, Kosmas C, Dokou A, Felekouras E, Antoniou E, Polyzos A, Sarantonis J, et al: Predictors of survival in stage IV metastatic colorectal cancer. Anticancer Res 30: 653-660, 2010.

11. Garcia MA, Nelson WJ and Chavez N: Cell-cell junctions organize structural and signaling networks. Cold Spring Harb Perspect Biol 10: a029181, 2018.

12. Anderson JM and Van Itallie CM: Physiology and function of the tight junction. Cold Spring Harb Perspect Biol 1: a002584, 2009.

13. Martin TA: The role of tight junctions in cancer metastasis. Semin Cell Dev Biol 36: 224-231, 2014.

14. Schuetz A, Radusheva V, Krug SM and Heinemann U: Crystal structure of the tricellulin C-terminal coiled-coil domain reveals a unique mode of dimerization. Ann NY Acad Sci 1405: 147-159, 2017.

15. Oda Y, Otani T, Ikenouchi J and Furuse M: Tricellulin regulates junctional tension of epithelial cells at tricellular contacts through Cdc42. J Cell Sci 127: 4201-4212, 2014.

16. Morampudi V, Graef FA, Stahl M, Dalwadi U, Conlin VS, Huang T, Vallance BA, Yu HB and Jacobson K: Tricellular tight junction protein tricellulin is targeted by the enteropathogenic escherichia coli effector EspG1, leading to epithelial barrier disruption. Infect Immun 85: e00700-16, 2016.

17. Takasawa A, Murata M, Takasawa K, Ono Y, Osanai M, Tanaka S, Nojima M, Kono T, Hirata K, Kojima T and Sawada N: Nuclear localization of tricellulin promotes the oncogenic property of pancreatic cancer. Sci Rep 6: 33582, 2016.
18. Benson AB III, Venook AP, Cederquist L, Chan E, Chen YJ, Cooper HS, Deming D, Engstrom PF, Enzinger PC, Fichera A, et al: Colon cancer, version 1.2017, NCCN clinical practice guidelines in oncology. J Natl Compr Canc Netw 15: 370-398, 2017.

19. Yang F, Xu J, Li H, Tan M, Xiong X and Sun Y: FBXW2 suppresses migration and invasion of lung cancer cells via promoting $\beta$-catenin ubiquitylation and degradation. Nat Commun 10: 1382, 2019.

20. Sadeghi MR, Jeddi F, Soozangar N, Somi MH, Shirmohamadi M, Khaze V and Samadi N: Nrf2/P-glycoprotein axis is associated with clinicopathological characteristics in colorectal cancer. Biomed Pharmacother 104: 458-464, 2018.

21. Hwang JY, Park JH, Kim MJ, Kim WJ, Ha KT, Choi BT, Lee SY and Shin HK: Isolinderalactone regulates the BCL-2/caspase-3/PARP pathway and suppresses tumor growth in a human glioblastoma multiforme xenograft mouse model. Cancer Lett 443: 25-33, 2019.

22. Livak KJ and Schmittgen TD: Analysis of relative gene expression data using real-time quantitative PCR and the 2(-Delta Delta C(T)) method. Methods 25: 402-408, 2001.

23. Qin M, Zhang J, Xu C, Peng P, Tan L, Liu S and Huang J: Knockdown of NIK and IKK $\beta$-binding protein (NIBP) reduces colorectal cancer metastasis through down-regulation of the canonical NF- $\mathrm{kB}$ signaling pathway and suppression of MAPK signaling mediated through ERK and JNK. PLoS One 12: e0170595, 2017.

24. Ting L, Rad R, Gygi SP and Haas W: MS3 eliminates ratio distortion in isobaric multiplexed quantitative proteomics. Nat Methods 8: 937-940, 2011.

25. Tolonen AC and Haas W: Quantitative proteomics using reductive dimethylation for stable isotope labeling. J Vis Exp: 51416, 2014.

26. Wang K, Shan Z, Duan L, Gong T, Liu F, Zhang Y, Wang Z, Shen J and Lei L: iTRAQ-based quantitative proteomic analysis of Yamanaka factors reprogrammed breast cancer cells. Oncotarget 8: 34330-34339, 2017.

27. Ashburner M, Ball CA, Blake JA, Botstein D, Butler H, Cherry JM, Davis AP, Dolinski K, Dwight SS, Eppig JT, et al: Gene ontology: Tool for the unification of biology. The gene ontology consortium. Nat Genet 25: 25-29, 2000.

28. Kanehisa M, Sato Y, Kawashima M, Furumichi M and Tanabe M: KEGG as a reference resource for gene and protein annotation. Nucleic Acids Res 44: D457-D462, 2016.

29. Szklarczyk D, Franceschini A, Wyder S, Forslund K, Heller D, Huerta-Cepas J, Simonovic M, Roth A, Santos A, Tsafou KP, et al: STRING v10: Protein-protein interaction networks, integrated over the tree of life. Nucleic Acids Res 43: D447-D452, 2015.

30. Zhao GX, Xu YY, Weng SQ, Zhang S, Chen Y, Shen XZ, Dong L and Chen S: CAPS1 promotes colorectal cancer metastasis via Snail mediated epithelial mesenchymal transformation. Oncogene 38: 4574-4589, 2019.

31. Xu CY, Qin MB, Tan L, Liu SQ and Huang JA: NIBP impacts on the expression of E-cadherin, CD44 and vimentin in colon cancer via the NF-кB pathway. Mol Med Rep 13: 5379-5385, 2016.

32. Zeisel MB, Dhawan P and Baumert TF: Tight junction proteins in gastrointestinal and liver disease. Gut 68: 547-561, 2019.

33. France MM and Turner JR: The mucosal barrier at a glance. J Cell Sci 130: 307-314, 2017.

34. Pastushenko I and Blanpain C: EMT transition states during tumor progression and metastasis. Trends Cell Biol 29: 212-226, 2019.

35. Gonzalez DM and Medici D: Signaling mechanisms of the epithelial-mesenchymal transition. Sci Signal 7: re8, 2014.

36. Xu CY, Liu SQ, Qin MB, Zhuge CF, Qin L, Qin N, Lai MY and Huang JA: SphK1 modulates cell migration and EMT-related marker expression by regulating the expression of p-FAK in colorectal cancer cells. Int J Mol Med 39: 1277-1284, 2017.

37. Ikenouchi J, Furuse M, Furuse K, Sasaki H, Tsukita S and Tsukita S: Tricellulin constitutes a novel barrier at tricellular contacts of epithelial cells. J Cell Biol 171: 939-945, 2005.

38. Shimada H, Satohisa S, Kohno T, Takahashi S, Hatakeyama T, Konno T, Tsujiwaki M, Saito T and Kojima T: The roles of tricellular tight junction protein lipolysis-stimulated lipoprotein receptor in malignancy of human endometrial cancer cells. Oncotarget 7: 27735-27752, 2016.

39. Schlachter K, Gyugos M, Halasz J, Lendvai G, Baghy K, Garami M, Gyöngyösi B, Schaff Z and Kiss A: High tricellulin expression is associated with better survival in human hepatoblastoma. Histopathology 65: 631-641, 2014. 
40. Masuda R, Semba S, Mizuuchi E, Yanagihara K and Yokozaki $\mathrm{H}$ Negative regulation of the tight junction protein tricellulin by snail-induced epithelial-mesenchymal transition in gastric carcinoma cells. Pathobiology 77: 106-113, 2010.

41. Somorácz A, Korompay A, Törzsök P, Patonai A, Erdélyi-Belle B, Lotz G, Schaff Z and Kiss A: Tricellulin expression and its prognostic significance in primary liver carcinomas. Pathol Oncol Res 20: 755-764, 2014.

42. Naugler WE and Karin M: NF-kappaB and cancer-identifying targets and mechanisms. Curr Opin Genet Dev 18: 19-26, 2008.

43. Gilmore TD: Introduction to NF-kappaB: Players, pathways, perspectives. Oncogene 25: 6680-6684, 2006.

44. Escarcega RO, Fuentes-Alexandro S, Garcia-Carrasco M, Gatica A and Zamora A: The transcription factor nuclear factor-kappa B and cancer. Clin Oncol (R Coll Radiol) 19: 154-161, 2007.
45. Kojima T, Fuchimoto J, Yamaguchi H, Ito T, Takasawa A, Ninomiya T, Kikuchi S, Ogasawara N, Ohkuni T, Masaki T, et al: $\mathrm{c}$-Jun N-terminal kinase is largely involved in the regulation of tricellular tight junctions via tricellulin in human pancreatic duct epithelial cells. J Cell Physiol 225: 720-733, 2010.

46. Xia Y, Shen S and Verma IM: NF- $\kappa$ B, an active player in human cancers. Cancer Immunol Res 2: 823-830, 2014.

This work is licensed under a Creative Commons Attribution-NonCommercial-NoDerivatives 4.0 International (CC BY-NC-ND 4.0) License. 